\title{
Multifractal cross wavelet analysis
}

\author{
Zhi-Qiang Jiang, ${ }^{1,2,3}$ Xing-Lu Gao, ${ }^{1}$ Wei-Xing Zhou,,${ }^{1,2,4, \text { * } \text { and H. Eugene Stanley }}{ }^{3}$ \\ ${ }^{1}$ Department of Finance, East China University of Science and Technology, Shanghai 200237, China \\ ${ }^{2}$ Research Center for Econophysics, East China University of Science and Technology, Shanghai 200237, China \\ ${ }^{3}$ Department of Physics and Center for Polymer Studies, Boston University, Boston, MA 02215, USA \\ ${ }^{4}$ Department of Mathematics, East China University of Science and Technology, Shanghai 200237, China
}

(Dated: February 27, 2018)

Complex systems are composed of mutually interacting components and the output values of these components usually exhibit long-range cross-correlations. Using wavelet analysis, we propose a method of characterizing the joint multifractal nature of these long-range cross correlations, a method we call multifractal cross wavelet analysis (MFXWT). We assess the performance of the MFXWT method by performing extensive numerical experiments on the dual binomial measures with multifractal cross correlations and the bivariate fractional Brownian motions (bFBMs) with monofractal cross correlations. For binomial multifractal measures, we find the empirical joint multifractality of MFXWT to be in approximate agreement with the theoretical formula. For bFBMs, MFXWT may provide spurious multifractality because of the wide spanning range of the multifractal spectrum. We also apply the MFXWT method to stock market indices, and in pairs of index returns and volatilities we find an intriguing joint multifractal behavior. The tests on surrogate series also reveal that the cross correlation behavior, particularly the cross correlation with zero lag, is the main origin of cross multifractality.

Keywords: Joint multifractal analysis; wavelet analysis; binomial measure; bivariate fractional Brownian motion; bootstrap. 


\section{INTRODUCTION}

In recent years a series of multifractal cross-correlation analysis methods have been developed and applied to a number of different fields. The goal has been to unveil possible multifractal long-range cross correlations between two time series. Such long-range cross correlations in pairs of series have widely applied in financial markets, ranging from uncovering the facts of cross multifractal nature 1 3] in different markets to building trading strategies to get excess returns [4, from improving the estimation of hedge ratio [5] to incorporating the copula-multifractality into the calculation of volatilities [6]. An early method, joint multifractal analysis, was invented in 1990 to study the relationship between the dissipation rates of kinetic energy and passive scalar fluctuations in fully developed turbulence and to handle the joint partition function of two multifractal measures [7]. This method is also referred to as the multifractal cross-correlation analysis based on the partition function approach (MFXPF) [8]. A special case of MFXPF, multifractal statistical moment cross-correlation analysis (MFSMXA), was independently invented in 2012 to study volatility time series in finance 9. In 2015, the main properties of the joint multifractal nature of binomial measures were derived and numerically validated [8].

Another multifractal cross-correlation analysis method is multifractal height cross-correlation analysis (MF-HXA) [10], which is a bivariate generalization of height-height correlation analysis [11. The MF-HXA method also has its origin in turbulence and is an extension of the cross-correlation analysis of the structure functions of temperature and velocity dissipation fields in a heated turbulent jet [12. Hence it is also a multifractal cross-correlation analysis based on structure function (MFXSF).

Other multifractal cross-correlation analysis methods include multifractal detrended cross-correlation analysis based on detrended fluctuation analysis (MFXDFA) [13, which is a multifractal version of detrended cross-correlation analysis (DCCA) [14, multifractal detrended cross-correlation analysis based on detrending moving-average analysis (MFXDMA) 15 based on multifractal detrending moving-average analysis (MF-DMA) 16] and detrending movingaverage analysis (DMA) [17. 24, multifractal cross-correlation analysis (MFCCA) [25, 26], and multifractal detrended partial correlation analysis (MFDPXA) [27.

Wavelet transform has long been applied to the study of fractals and multifractals [28, 29] and a partition function approach based on wavelet transform has been proposed [30. Here we generalize multifractal wavelet analysis to the bivariate case and propose a new joint multifractal analysis based on the wavelet transform of two time series, which is a multifractal generalization of the cross wavelet transform 31-33. We thus can also call it multifractal cross wavelet analysis (MFXWT). Similar to when we use the MFXPF method, we introduce two orders in MFXWT. We test the validity of the method by conducting numerical experiments with two mathematical models and gain explicit analytical results. Finally we apply the method to an empirical time series.

The rest of paper is organized as follows. In Section 2, we present a framework of the MFXWT approach. Extensive numerical experiments using binomial measures and bivariate fractional Brownian motions with known analytical multifracal expressions are conducted in Section 3 to check the validity of MFXWT approach. In Section 4 we apply the MFXWT algorithms to the pair of daily returns, as well as the pair of daily volatilities. Statistical tests indicate that the MFXWT method has the ability to detect the cross multifractality in pairs of financial series. Section 5 concludes.

\section{METHODS}

Following Refs. 34, 35, we define the wavelet transform of a given time series $x(t)$ as

$$
w(s, i)=\frac{1}{s} \sum_{t=1}^{n} x(t) \psi[(t-i) / s], \quad i=1, \cdots, n
$$

where $\psi(x)$ is the wavelet kernel shifted by $i, s$ is the scale, and $n$ is the length of $x(t)$. We use the wavelet transform to decompose the signals in the time-scale plane. The resulting wavelet coefficients are an indicator of the singular behavior of signals when the wavelet kernel is $\int x^{m+1} \psi(s) \mathrm{d} x=0$ [36], and from this we approximate the signal trends by polynomials up to order $m$. A good choice of $\psi(x)$ is derivative $m$ of a Gaussian, $\psi^{m}(x)=\mathrm{d}^{m}\left(e^{-x^{2} / 2}\right) / \mathrm{d} x^{m}$. Here we use the "Mexican hat" $m=2$.

Using the wavelet-based scaling (or multiscaling) estimator [37, 38, and cross correlation (or multifractal) analysis 8 10, 13 15, we propose a new method for detecting the multifractal cross correlations in a pair of series $x(t)$ and $y(t)$ based on wavelet analysis, i.e., a multifractal cross wavelet analysis with two moment orders (MFXWT $(p, q))$.

We first perform a wavelet transform of the two time series and obtain the wavelet coefficients $w_{x}(s, i)$ and $w_{y}(s, i)$. 
We then define the joint partition function with moments $p$ and $q$ based on the obtained wavelet coefficients,

$$
\chi_{x y}(p, q, s)=\sum_{i=1}^{n}\left|w_{x}(s, i)\right|^{p / 2}\left|w_{y}(s, i)\right|^{q / 2} .
$$

Because some wavelet coefficients approach 0 , the partition function diverges for $p<0$ or $q<0$. When $w_{x}=w_{y}$ and $p=q$, we use wavelet analysis to recover the traditional partition function. The part $\left|w_{x}(s, i)\right|^{p / 2}\left|w_{y}(s, i)\right|^{q / 2}$ is a generalized cross wavelet spectrum and it recovers the traditional cross wavelet spectrum when $p=4$ and $q=4$ [39], as the wavelet coefficients are real number. The cross wavelet spectrum can be used to calculate the wavelet coherency, which is able to uncover the co-movement between two series in the time-frequency domain [40, 41]. The definition of the partition function allows us to uncover the more intricate relationship between the coherency and the scale under different scopes, which corresponds to the cross multifractal behaviors. If the underlying processes are jointly multifractal, the result is a scaling behavior,

$$
\chi_{x y}(p, q, s) \sim s^{\mathcal{T}_{x y}(p, q)} .
$$

where $\mathcal{T}_{x y}(p, q)$ is the joint mass exponent function. Note that we can estimate $\mathcal{T}_{x y}(p, q)$ by regressing $\ln \chi_{x y}(p, q, s)$ against $\ln s$ in the scaling range for a given pair $(p, q)$.

Analogous to the double Legendre transforms in the joint multifractal analysis based on the partition function approach $\operatorname{MFXPF}(p, q)$ [], we define the joint singularity strength function $h_{x}$ and $h_{y}$

$$
\begin{aligned}
& h_{x}(p, q)=2 \partial \mathcal{T}_{x y}(p, q) / \partial p \\
& h_{y}(p, q)=2 \partial \mathcal{T}_{x y}(p, q) / \partial q,
\end{aligned}
$$

and the multifractal spectrum $D_{x y}\left(h_{x}, h_{y}\right)$

$$
D_{x y}\left(h_{x}, h_{y}\right)=p h_{x} / 2+q h_{y} / 2-\mathcal{T}_{x y}
$$

The values $h_{x}(p, q), h_{y}(p, q)$, and $D_{x y}\left(h_{x}, h_{y}\right)$ from the $\operatorname{MFXWT}(p, q)$ method differ from the joint singularity strengths $\alpha_{x}(p, q), \alpha_{y}(p, q)$ and the joint multifractal spectrum $f_{x y}\left(\alpha_{x}, \alpha_{y}\right)$ obtained from the $\operatorname{MFXPF}(p, q)$ method. For example, when $p=0$ and $q=0$ the joint partition function in Eq. (2) is equal to the number of wavelet coefficients and corresponds to the total number of data points in the original series, which means that $\mathcal{T}_{x y}(0,0)=0$ and that in the $\operatorname{MFXPF}(p, q)$ method $\tau_{x y}(0,0)=-1$. We also find that all the estimated $h_{x}$ and $h_{y}$ are less than 0 . Although this violates our intuition that the singularity strength should be positive, these differences in value do not mean that our method is useless because the joint multifractal quantities obtained from both methods still share the same physical meanings and geometric features, which allows us to determine the cross correlations in the time series pairs. Following the usual numerical experiments and empirical analysis in which the multifractal analysis based wavelet estimators are performed on integral series, we also test our $\operatorname{MFXWT}(p, q)$ method on integral series. The obtained results are not easy to explain, however, and it is very difficult to link to the theoretical values in the $p$-model [42]. Thus we focus our investigations on non-cumulative series, e.g., stock returns rather than stock prices.

Reference [30] found errors in the estimation of joint singularity strength and joint multifractal spectrum based on the Legendre transform. They proposed an alternative method of computing $h$ and $D(h)$ using the direct estimation canonical method. Similarly we directly estimate the joint singularity strength $h_{x}$ and $h_{y}$ and the joint multifractal spectrum $D_{x y}(p, q)$ using

$$
\begin{aligned}
h_{x}(p, q) & =\lim _{s \rightarrow 0} \frac{1}{\ln s} \sum_{i} \mu_{x y}(p, q, s, i) \ln \left|w_{x}(s, i)\right|, \\
h_{y}(p, q) & =\lim _{s \rightarrow 0} \frac{1}{\ln s} \sum_{i} \mu_{x y}(p, q, s, i) \ln \left|w_{y}(s, i)\right|, \\
D_{x y}(p, q) & =\lim _{s \rightarrow 0} \frac{1}{\ln s} \sum_{i} \mu_{x y}(p, q, s, i) \ln \mu_{x y}(p, q, s, i) .
\end{aligned}
$$

where

$$
\mu_{x y}(p, q, s, i)=\left|w_{x}(s, t)\right|^{p / 2}\left|w_{y}(s, i)\right|^{q / 2} / \chi_{x y}(p, q, s) .
$$

Thus we can directly determine the joint singularity strength functions $h_{x}(p, q)$ and $h_{y}(p, q)$ and the joint multifractal function $D_{x y}(p, q)$ from Eqs. 79 . 

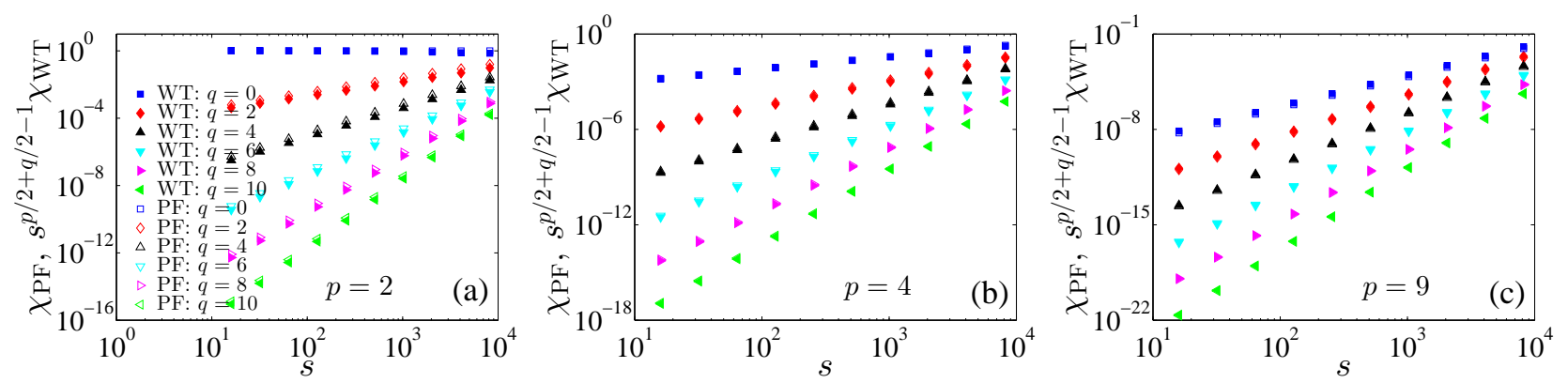

FIG. 1. (Color online) Comparison of the scaling behaviors of two binomial measures for the joint partition functions obtained from $\operatorname{MFXPF}(p, q)$ and $\operatorname{MFXWT}(p, q)$ with different values of $p$ and $q$. In the plots, $q$ varies from 0 to 10 with a step of 2 . The joint partition functions of $\operatorname{MFXWT}(p, q)$ are scaled by a factor of $s^{p / 2+q / 2-1}$, which results in the almost same scaling pattern as the joint partition functions of $\operatorname{MFXPF}(p, q)$.

\section{NUMERICAL EXPERIMENTS}

To test the validity and performance of the proposed MFXWT $(p, q)$ method we conduct two binomial measurements generated (i) from the multiplicative $p$-model [42] and from (ii) bivariate fractional Brownian motions (bFBMs) [4345].

\section{A. Binomial measures}

We first numerically test the validity of the $\operatorname{MFXWT}(p, q)$ method using two binomial measures from the $p$-model with known analytic multifractal properties, (i) $\left\{x(i): i=1,2, \cdots, 2^{k}\right\}$ and (ii) $\left\{y(i): i=1,2, \cdots, 2^{k}\right\}$ [42]. Each binomial measure is generated iteratively. We start with the zeroth iteration $k=0$, where the data set $z(i)$ consists of one value, $z^{(0)}(1)=1$. In iteration $k$, the data set $\left\{z^{(k)}(i): i=1,2, \cdots, 2^{k}\right\}$ is obtained from

$$
\begin{aligned}
& z^{(k)}(2 i-1)=p_{z} z^{(k-1)}(i) \\
& z^{(k)}(2 i)=\left(1-p_{z}\right) z^{(k-1)}(i)
\end{aligned}
$$

for $i=1,2, \cdots, 2^{k-1}$. When $k \rightarrow \infty, z^{(k)}(i)$ approaches a binomial measure with a scaling exponent function $H_{z z}(q)$ and a mass exponent function $\tau_{z z}(q)$ that have an analytic form [42, 46]

$$
\begin{aligned}
H_{z z}(q) & =1 / q-\log _{2}\left[p_{z}^{q}+\left(1-p_{z}\right)^{q}\right] / q, \\
\tau_{z z}(q) & =-\log _{2}\left[p_{z}^{q}+\left(1-p_{z}\right)^{q}\right] .
\end{aligned}
$$

In our numerical experiment, the parameters of the two binomial measures from the $p$-model are set at $p_{x}=0.3$ for $x(i)$ and $p_{y}=0.4$ for $y(i)$ with an iterative step $k=16$. The analytic scaling exponent functions $H_{x x}(q)$ and $H_{y y}(q)$ of $x$ and $y$ are shown in Eq. (11). Because the two series are generated using the same rule, the two series $x$ and $y$ exhibit a strong correlation with a coefficient of 0.82 .

Xie et al. analytically derived the joint multifractal properties for two binomial measures constructed from the $p$-model [8]. The joint mass exponent function $\tau_{x y}(p, q)$,

$$
\tau_{x y}(p, q)=\frac{p \gamma}{2 \ln 2}-\frac{\ln \left[p_{y}^{Q}+\left(1-p_{y}\right)^{Q}\right]}{\ln 2},
$$

the two joint singularity strength functions $\alpha_{x}(p, q)$ and $\alpha_{y}(p, q)$,

$$
\begin{gathered}
\alpha_{x}(p, q)=\frac{\gamma}{\ln 2}-\frac{\beta}{\ln 2} \frac{p_{y}^{Q} \ln p_{y}+\left(1-p_{y}\right)^{Q} \ln \left(1-p_{y}\right)}{p_{y}^{Q}+\left(1-p_{y}\right)^{Q}}, \\
\alpha_{y}(p, q)=-\frac{1}{\ln 2} \frac{p_{y}^{Q} \ln p_{y}+\left(1-p_{y}\right)^{Q} \ln \left(1-p_{y}\right)}{p_{y}^{Q}+\left(1-p_{y}\right)^{Q}},
\end{gathered}
$$



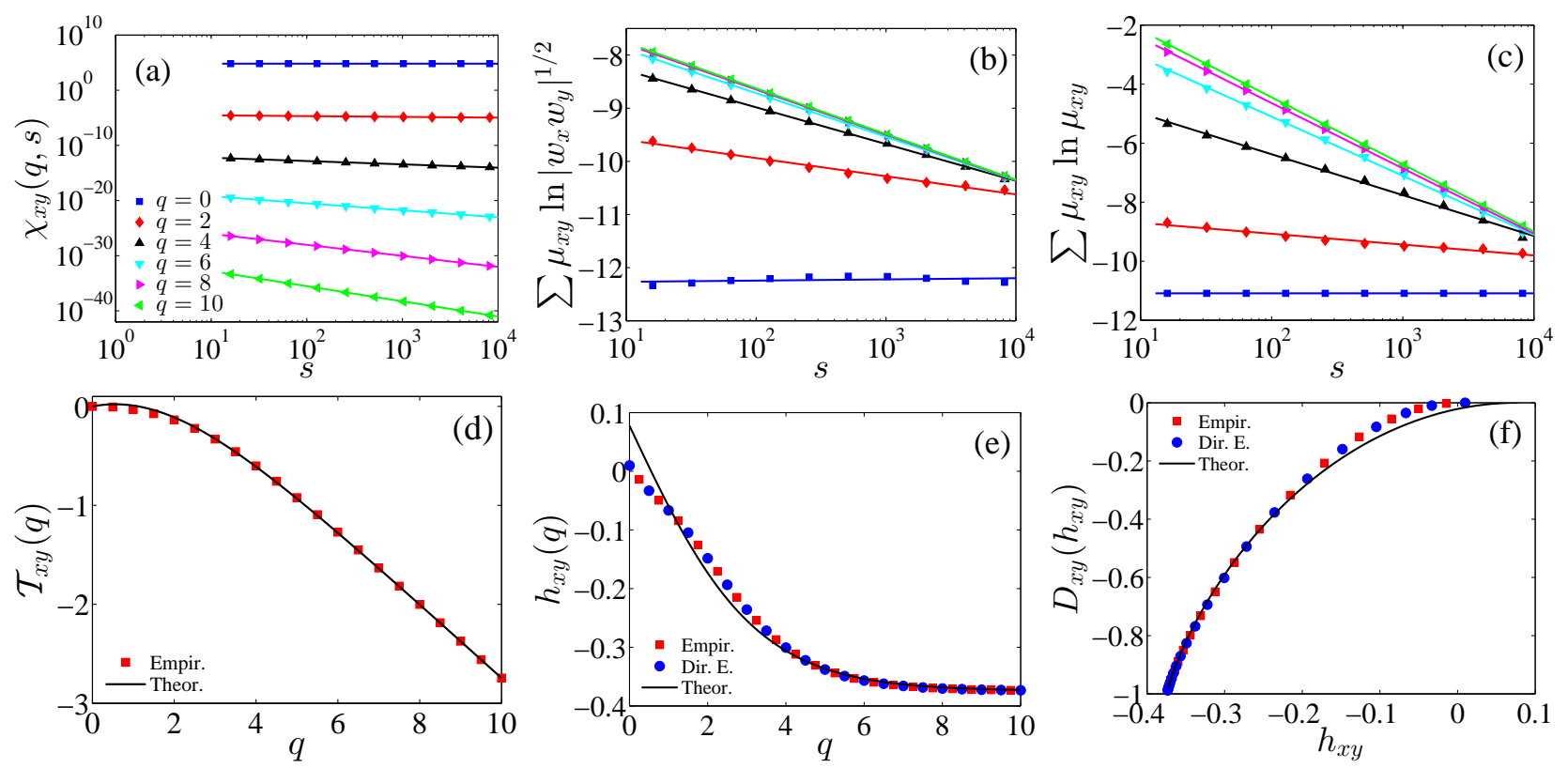

FIG. 2. (Color online) Multifractal cross wavelet analysis of two binomial measures with $p_{x}=0.3$ and $p_{y}=0.4$ based on the $\operatorname{MFXWT}(q)$ method. (a) Power-law behaviors between $\chi_{x y}(q, s)$ and the scale $s$ for different $q$. (b) Linear relationship of $\sum_{t} \mu_{x y}(q, s, t) \ln \left|w_{x}(s, t) w_{y}(s, t)\right|^{1 / 2}$ against ln $s$. (c) Linear relationship of $\sum_{t} \mu_{x y}(q, s, t) \ln \mu_{x y}(q, s, t)$ with respect to ln $s$. (d) Joint mass exponent function $\mathcal{T}_{x y}(q)$. (e) Joint singularity strength function $h_{x y}(q)$. (f) Joint multifractal singularity spectrum $D_{x y}\left(h_{x y}\right)$.

and the joint multifractal spectrum $f_{x y}(p, q)$ is expressed as

$$
f_{x y}\left(\alpha_{x}, \alpha_{y}\right)=\frac{Q Z^{Q} \ln Z+\left(1+Z^{Q}\right) \ln \left(1+Z^{Q}\right)}{\ln 2\left(1+Z^{Q}\right)},
$$

where $\beta=\frac{\ln p_{x}-\ln \left(1-p_{x}\right)}{\ln p_{y}-\ln \left(1-p_{y}\right)}, \gamma=\beta \ln \left(1-p_{y}\right)-\ln \left(1-p_{x}\right), Q=\beta p / 2+q / 2$, and $Z=\frac{1-p_{y}}{p_{y}}$. These theoretical formulas have been found to numerically agree with the empirical results from the $\operatorname{MFXPF}(p, q)$ method $[8$, and this allows us to check whether these theoretical formulas can be employed as a benchmark test of the performance of the $\operatorname{MFXWT}(p, q)$ algorithm when it is applied to binomial measures. By comparing the scaling behaviors of the joint partition functions from both methods, we find the theoretical formulas of the joint mass exponent function $\mathcal{T}_{x y}(p, q)$, the joint singularity strength functions $h_{x}(p, q)$ and $h_{y}(p, q)$, and the joint multifractal spectrum $D_{x y}\left(h_{x}, h_{y}\right)$ for $\operatorname{MFXWT}(p, q)$.

Figure 1 shows the scaling behavior of the joint partition functions obtained from the $\operatorname{MFXPF}(p, q)$ and $\operatorname{MFXWT}(p, q)$ methods with different values of $p$ and $q$. The joint partition functions of $\operatorname{MFXWT}(p, q)$ are scaled by a factor of $s^{p / 2+q / 2-1}$. We find that there is a slightly difference between the markers of both methods in panel (a) and such differences disappeared in panel (b) and (c). This indicates almost the same scaling behavior between the scaled joint partition functions of $\operatorname{MFXWT}(p, q)$ and the joint partition functions of $\operatorname{MFXPF}(p, q)$, which allow us to connect the theoretical joint multifractal formulas of binomial measures to the empirical joint multifractal features of $\operatorname{MFXWT}(p, q)$ by using

$$
\begin{aligned}
\mathcal{T}_{x y}(p, q)+p / 2+q / 2-1 & =\tau_{x y}(p, q), \\
h_{x}(p, q)+1 & =\alpha_{x}(p, q), \\
h_{y}(p, q)+1 & =\alpha_{y}(p, q), \\
D_{x y}\left(h_{x}, h_{y}\right)+1 & =f_{x y}\left(\alpha_{x}, \alpha_{y}\right),
\end{aligned}
$$

where $\tau_{x y}(p, q), \alpha_{x}(p, q), \alpha_{y}(p, q)$, and $f_{x y}\left(\alpha_{x}, \alpha_{y}\right)$ are given by Eqs. 1316$)$.

These formulas are an efficient test of the estimation accuracy of the $\operatorname{MFXWT}(p, q)$ method in the joint multifractal analysis of two binomial measures. Using the partition function approach and wavelet analysis to detect the multifractal nature of a single time series, $\tau_{x x}(q)=\mathcal{T}_{x x}(q)+q$ and $\alpha_{x}(q)=h_{x}(q)+1$ [47-49].

We first examine the case of $p=q$. Figure 2(a) shows the scaling behavior between the joint partition functions $\chi_{x y}(q, s)$ and the scale $s$. Note that there is a significant power-law dependence over more than three orders of 
magnitude. By estimating the power-law exponents between $\chi_{x y}(q, s)$ and $s$ for different $q$, we find the joint mass exponent function $\mathcal{T}(q)$ [see the plot in Fig. 2(d)]. Figure2(d) also shows the theoretical values of $\mathcal{T}(q)$ obtained from Eq. 17). The two curves closely match, suggesting that our $\operatorname{MFXWT}(p, q)$ algorithm accurately analyzes the joint multifractal nature in two binomial measures. As expected, the nonlinear behavior of $\mathcal{T}(q)$ against $q$ also demonstrates the joint multifractality in binomial measures.

Figures 2 (b) and 2 (c) show the power-law scaling behaviors of two quantities $\left(\sum \mu_{x y} \ln \left|w_{x} w_{y}\right|^{1 / 2}\right.$ and $\left.\sum \mu_{x y} \ln \mu_{x y}\right)$ against the scale $s$, whose power-law exponents are estimates of the joint singularity strength $h_{x y}$ and the joint multifractal function $D\left(h_{x y}\right)$.
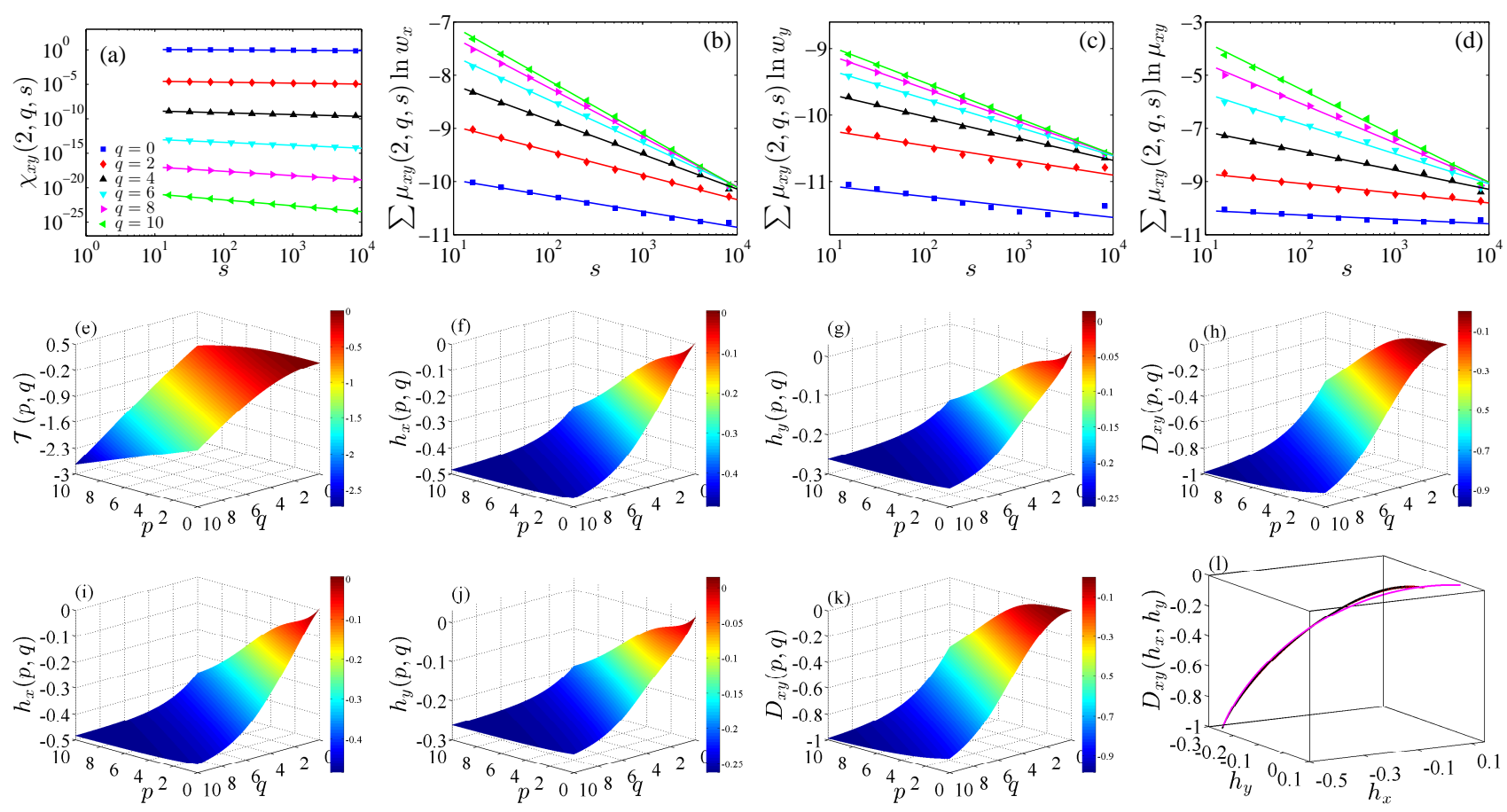

FIG. 3. (Color online) Multifractal cross wavelet transform analysis of two binomial measures with $p_{x}=0.3$ and $p_{y}=0.4$ based on the bi-order $\operatorname{MFXWT}(p, q)$ method. (a) Power-law plots of $\chi_{x y}(p, q, s)$ with respect to the scale $s$ for different $q$ with fixed $p=2$. (b) Linear dependence of $\sum_{t} \mu_{x y}(2, q, s, t) \ln \left|w_{x}(s, t)\right|$ against $\ln s$ for different $q$ with fixed $q=2$. (c) Linear dependence of $\sum_{t} \mu_{x y}(2, q, s, t) \ln \left|w_{y}(s, t)\right|$ against $\ln s$ for different $q$ with fixed $q=2$. (d) Linear dependence of $\sum_{t} \mu_{x y}(2, q, s, t) \ln \mu_{x y}(2, q, s, t)$ against $\ln s$ for different $q$ with fixed $q=2$. (e)-(h) Joint mass exponent function $(\mathcal{T})_{x y}(p, q)$, joint singularity functions $h_{x}(p, q)$ and $h_{y}(p, q)$, and joint multifractal function $D_{x y}(p, q)$ from Eqs. (3-6). (i)-(k) Joint singularity functions $h_{x}(p, q)$ and $h_{y}(p, q)$ and joint multifractal function $D_{x y}(p, q)$ from Eqs. (79.9). (l) Joint multifractal spectrum $D_{x y}\left(h_{x}, h_{y}\right)$.

Figure 2(e) compares the joint singularity strength $h_{x y}(q)$ obtained using different methods. The solid line corresponds to theoretical values. The squares and circles are obtained from the first derivation of the joint mass exponent $\mathcal{T}_{x y}(q)$ and the direct estimating method, respectively. Note that although the empirical $h_{x y}(q)$ of both methods of estimating coincide, both empirical curves agree with the theoretical values only when $q \geq 1$. We see deviations when $q<1$, and the reason for this is not clear.

Figure 2(f) shows the corresponding joint multifractal spectra of binomial measures in both theoretical values and estimated values. The $D_{x y}\left(h_{x y}\right)$ values obtained from Eqs. (6) and (9) agree and collapse on the theoretical curves. Our results suggest that the accuracy of the $\operatorname{MFXWT}(p, q)$ is acceptable for analyzing the joint multifractality in binomial measures.

Releasing the $p=q$ restriction in Fig. 2, Fig. 3(a) shows how the joint partition function $\chi_{x y}(2, q, s)$ depends on the scale $s$ for different $q$ with fixed $p=2$. We see power-law behaviors. For each pair of $(p, q)$, the slope of the straight line in an estimate of the corresponding joint mass exponent $\mathcal{T}_{x y}(p, q)$. Figure 3(e) shows a plot of the joint mass exponent function $\mathcal{T}_{x y}(p, q)$ with respect to $p$ and $q$. Note that again there are nonlinear features between $\mathcal{T}_{x y}(p, q)$ and $(p, q)$, and this verifies joint multifractality in the two binomial measures. Following Eqs. (446), if we have the mass exponent $\mathcal{T}_{x y}(p, q)$ we can numerically compute the joint singularity strength functions $h_{x}(p, q)$ and $h_{y}(p, q)$ and the joint multifractal function $D_{x y}(p, q)$. Figures $3(\mathrm{f}), 3(\mathrm{~g})$ and $3(\mathrm{~h})$ show the corresponding $h_{x}(p, q), h_{y}(p, q)$, and $D_{x y}(p, q)$, respectively. The wide spanning range of $h_{x}, h_{y}$, and $D_{x y}$ further corroborates the joint multifractality in 
binomial measures.

The direct estimation method presented in Eqs. (7,9) is an alternative way to estimate the joint singularity strength $h_{x}(p, q)$ and $h_{y}(p, q)$ and the joint multifractal function $D_{x y}(p, q)$. By estimating the three quantities $\sum \mu_{x y} \ln \left|w_{x}\right|$, $\sum \mu_{x y} \ln \left|w_{y}\right|$, and $\sum \mu_{x y} \ln \mu_{x y}$ we find power-law scaling behaviors between these quantities and the scale $s$, as shown in Fig. 3(b-d). Their power-law exponents correspond to the joint singularity strength function $h_{x}(p, q)$ in Fig. $3(\mathrm{i})$ and $h_{y}(p, q)$ in Fig. $3(\mathrm{j})$ and the joint multifractal function $D_{x y}(p, q)$ in Fig. $3(\mathrm{k})$. Note that $h_{x}(p, q)$ in Fig. $3(\mathrm{f})$ and Fig. 3(i), $h_{y}(p, q)$ in Fig. 3(g) and Fig. 3(j), and $D_{x y}(p, q)$ in Fig. 3(h) and Fig. 3(k) obtained using both methods agree.
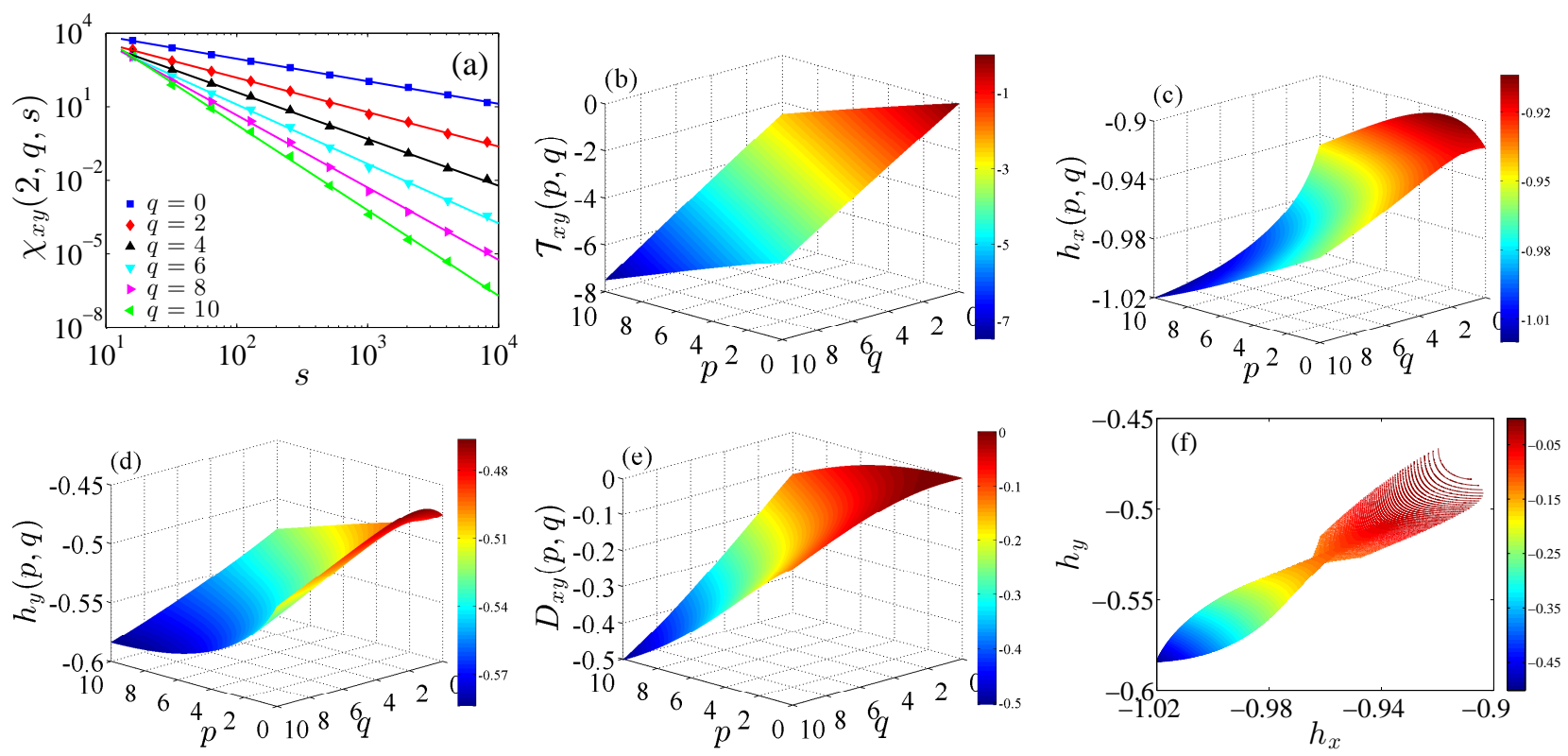

FIG. 4. (Color online) Multifractal cross wavelet analysis of bivariate fractional Brownian motion with $H_{x x}=0.1, H_{y y}=0.5$, and $\rho=0.5$. (a) Power-law relationship between $\chi_{x y}(p, q, s)$ and scale $s$ for fixed $p=2$ and different $q$. (b) Joint mass exponent function $\mathcal{T}_{x y}(p, q)$ obtained from Eq. (3). Using the least square method, we have $\mathcal{T}_{x y}=-0.485 p-0.268 q+0.135$. (c) Joint singularity function $h_{x}(p, q)$. (d) Joint singularity function $h_{y}(p, q)$. (e) Joint multifractal spectrum $D_{x y}(p, q)$. (f) Contour plots of joint multifractal spectrum $D_{x y}\left(h_{x}, h_{y}\right)$.

Figure 3(1) shows that both methods agree, and this illustrates the joint multifractal spectra $D_{x y}\left(h_{x}, h_{y}\right)$ of both methods, as well as the theoretical values (magenta curve) expressed in Eq. (16). Xie et al. [8] showed that the joint multifractal spectrum $f_{x y}\left(\alpha_{x}, \alpha_{y}\right)$ of binomial measures is a univariate function of $Q$, and thus also of $\alpha_{x}$ or of $\alpha_{y}$ due to Eqs. (14) and (15), and this means that the joint multifractal spectrum of MFXWT is also a univariate function of $h_{x}$ or of $h_{y}$ due to Eqs. (18) and (19). Figure 3(1) shows that the estimated joint multifractal spectra from both methods are a curve, not a surface, verifying the univariate function relationship between $D_{x y}$ and $h_{x}$ or $h_{y}$. Both estimated joint multifractal spectra approximately overlap with the theoretical multifractal spectrum, suggesting that the $\operatorname{MFXWT}(p, q)$ method provides an accurate joint multifractal analysis of binomial measures.

\section{B. Bivariate fractional Brownian motions (bFBMs)}

A bivariate fractional Brownian motion $[x(t), y(t)]$ with parameters $\left\{H_{x x}, H_{y y}\right\} \in(0,1)^{2}$ is a self-similar Gaussian process with stationary increments, where $x(t)$ and $y(t)$ are two univariate fractional Brownian motions with Hurst indices $H_{x x}$ and $H_{y y}$ and also are the two components of the bFBM [43 45. The basic properties of multivariate fractional Brownian motions have been extensively studied [43 45. Extensive numerical experiments of multifractal cross-correlation analysis algorithms have been performed on bFBMs [8, 15, 27. The two Hurst indices $H_{x x}$ and $H_{y y}$ of the two univariate FBMs and their cross-correlation coefficient $\rho$ are input arguments in the simulation algorithm. By using the simulation procedure described in Ref. [44, 45], we generate a realization of bFBM in which $H_{x x}=0.1$, $H_{y y}=0.5$, and $\rho=0.5$. The length of the bFBM is $2^{16}$.

As described in Ref. [8], if the two time series is monofractal, the joint singularity strengths $h_{x}(p, q)$ and $h_{y}(p, q)$ are constants, and their joint multifractal spectrum is $D_{x y}\left(h_{x}, h_{y}\right)=0$. Note that Eqs. 17 20 obtained from the $p$-model 
are no longer valid because they are derived using conservative measures, and the increments of both components $x(t)$ and $y(t)$ in bFBM are not conservative.

Figure 4 shows the results of the joint multifractal analysis of the bFBM using the MFXWT algorithm. Figure $4(\mathrm{a})$ shows how the joint partition functions $\chi_{x y}(2, q, s)$ of the wavelet coefficients are plotted with respect to the scale $s$ for fixed $p=2$ and different $q$. Again we see strong power-law scaling behaviors that allow us to estimate the joint mass exponents $\mathcal{T}_{x y}$ using the least square estimation method. Figure 4 (b) shows the joint mass exponent function against different $p$ and $q$. Because of the monofractality of the bFBMs, we see a plane for $\mathcal{T}(p, q)$. The bivariate regression yields

$$
\mathcal{T}_{x y}(p, q)=-0.485 p-0.268 q+0.135
$$

Using Eq. (6), we infer that $\bar{h}_{x}=-0.970, \bar{h}_{y}=-0.536$, and $\bar{D}_{x y}=-0.135$ deviate from the theoretical value $D_{x y}(0,0)=0$. When $p=q=0$, Eq. (21) gives $\mathcal{T}_{x y}(0,0)=0.135$, which also deviates from the theoretical value $\mathcal{T}_{x y}(0,0)=0$.
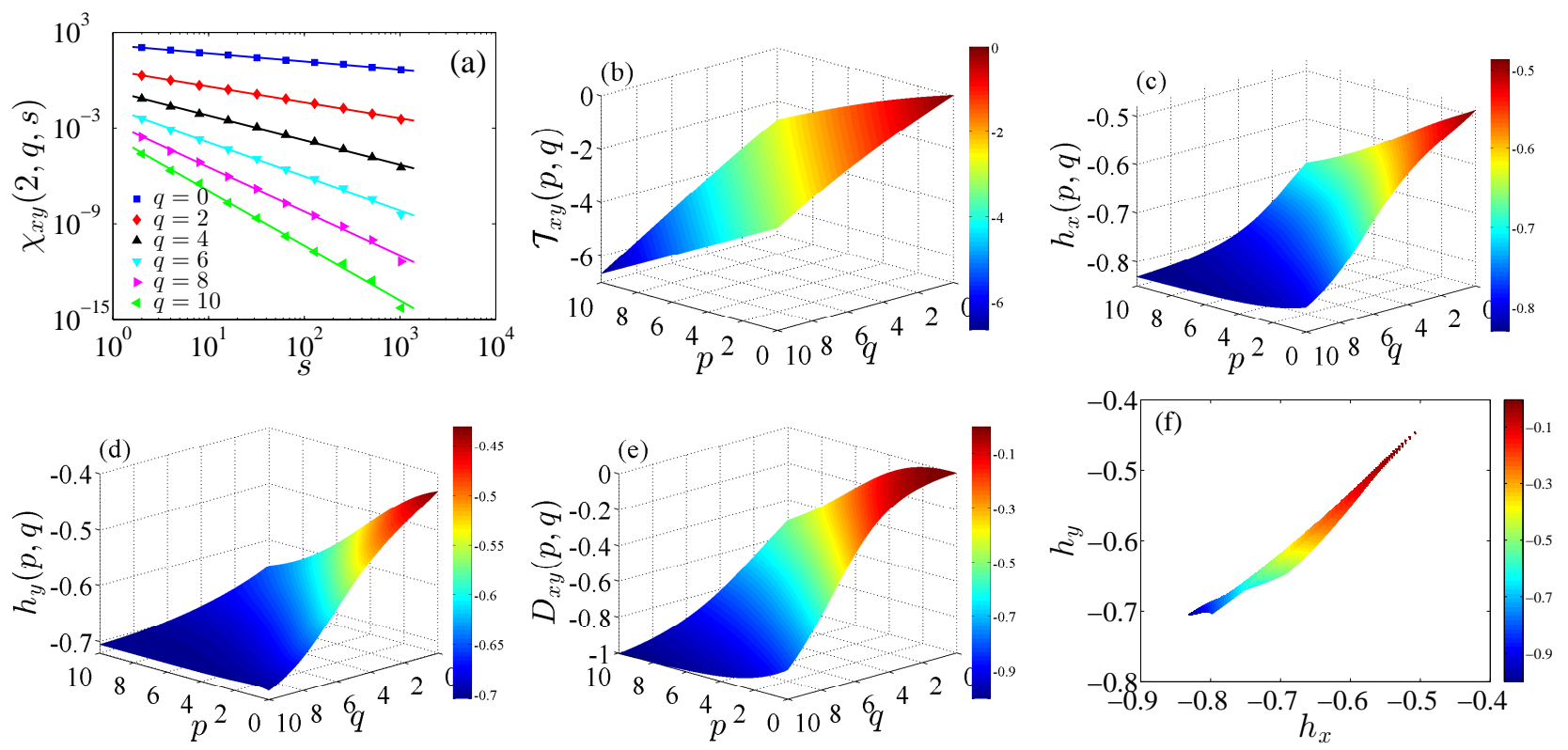

FIG. 5. (Color online) Multifractal cross wavelet analysis of the joint multifractality between the daily return series of DJIA index and NASDAQ index using the $\operatorname{MFXWT}(p, q)$ method. (a) Power-law dependence of $\chi_{x y}(p, q, s)$ on scale $s$ for fixed $p=2$ and different $q$. (b) Joint mass exponent function $\mathcal{T}(p, q)$. (c) Joint singularity strength function $h_{x}(p, q)$. (d) Joint singularity strength function $h_{y}(p, q)$. (e) Joint multifractal function $D_{x y}(p, q)$. (f) Joint multifractal singularity spectrum $D_{x y}\left(h_{x}, h_{y}\right)$.

Alternatively, using Eqs. (4) and (5) and a numerical differentiation of $\mathcal{T}_{x y}$, we can estimate $h_{x}(p, q)$ and $h_{y}(p, q)$. Figures 4(c) and 4(d) plot the estimated joint singularity strength functions $h_{x}(p, q)$ and $h_{y}(p, q)$ obtained from taking the forward difference of $\mathcal{T}_{x y}(p, q)$. Note that the singularity strength function $h_{x}(p, q)$ and $h_{y}(p, q)$ obtained from the numerical methods vary across a small range. The corresponding average value is -0.968 for $h_{x}$ and -0.534 for $h_{y}$, which agreea with $\bar{h}_{x}$ and $\bar{h}_{y}$ obtained from the plane equation of $\mathcal{T}_{x y}(p, q)$ in Eq. 211). Using the double Legendre transform in Eq. (6), we further obtain the joint multifractal function $D_{x y}$, which is plotted with respect to $p$ and $q$ in Fig. 4(e) and with respect to $h_{x}$ and $h_{y}$ in Fig. $4(\mathrm{f})$. The average value of $D_{x y}$ is -0.178 , also close to $\bar{D}_{x y}=-0.135$. However unlike $h_{x}$ and $h_{y}$, which span a narrow range, $D_{x y}$ spans a relatively wide range from 0 to 0.5. This indicates that the MFXWT method may indicate a spurious multifractality for bFBM if we determine the joint multifractality only within the spanning range of $D_{x y}$. This spurious multifractality often occurs when using the partition function approach and is usually caused by the finite size effect [50]. It suggests that we need to use bootstrapping to statistically test for the presence of multifractality [51, 52].

\section{APPLICATION TO STOCK MARKET INDICES}

We now apply the $\operatorname{MFXPF}(p, q)$ method to detect joint multifractality in the daily returns of the dow Jones industrial average (DJIA) and the National Association of Securities Dealers Automated Quotations (NASDAQ) 
index. To compare our results with those in Ref. [8], we also conduct a similar analysis on the volatility time series of the two indices. The daily return is defined as the logarithmic difference of daily closing price,

$$
R(t)=\ln I(t)-\ln I(t-1),
$$

where $I(t)$ is the closing price of the DJIA index or the NASDAQ index on day $t$. Both indices are retrieved from "Yahoo! Finance." The spanning period of both indices is from 5 February 1971 to 17 June 2016 and contain a total of 11430 data points. The volatilities are determined by the absolute values of the daily returns.

\section{A. Daily return time series}

We first analyze the joint multifractality of the daily returns of both indices using the $\operatorname{MFXWT}(p, q)$ method. Figure 5 shows the results.

Figure 5(a) plots the joint partition function $\chi_{x y}(2, q, s)$ as a function of the scale $s$ for fixed $p=2$ and varying $q$. We see strong power-law behavior in a scaling range larger than three orders of magnitude. The results for other $(p, q)$ pairs are similar. By regressing $\ln \chi_{x y}(p, q, s)$ with respect to $\ln s$ for a given pair of $(p, q)$, we obtain the joint mass exponents $\mathcal{T}_{x y}(p, q)$, which are plotted in Fig. 5(b). Note that the joint mass exponents are a nonlinear function of $p$ and $q$, indicating the presence of joint multifractality in the daily returns of the two indices.
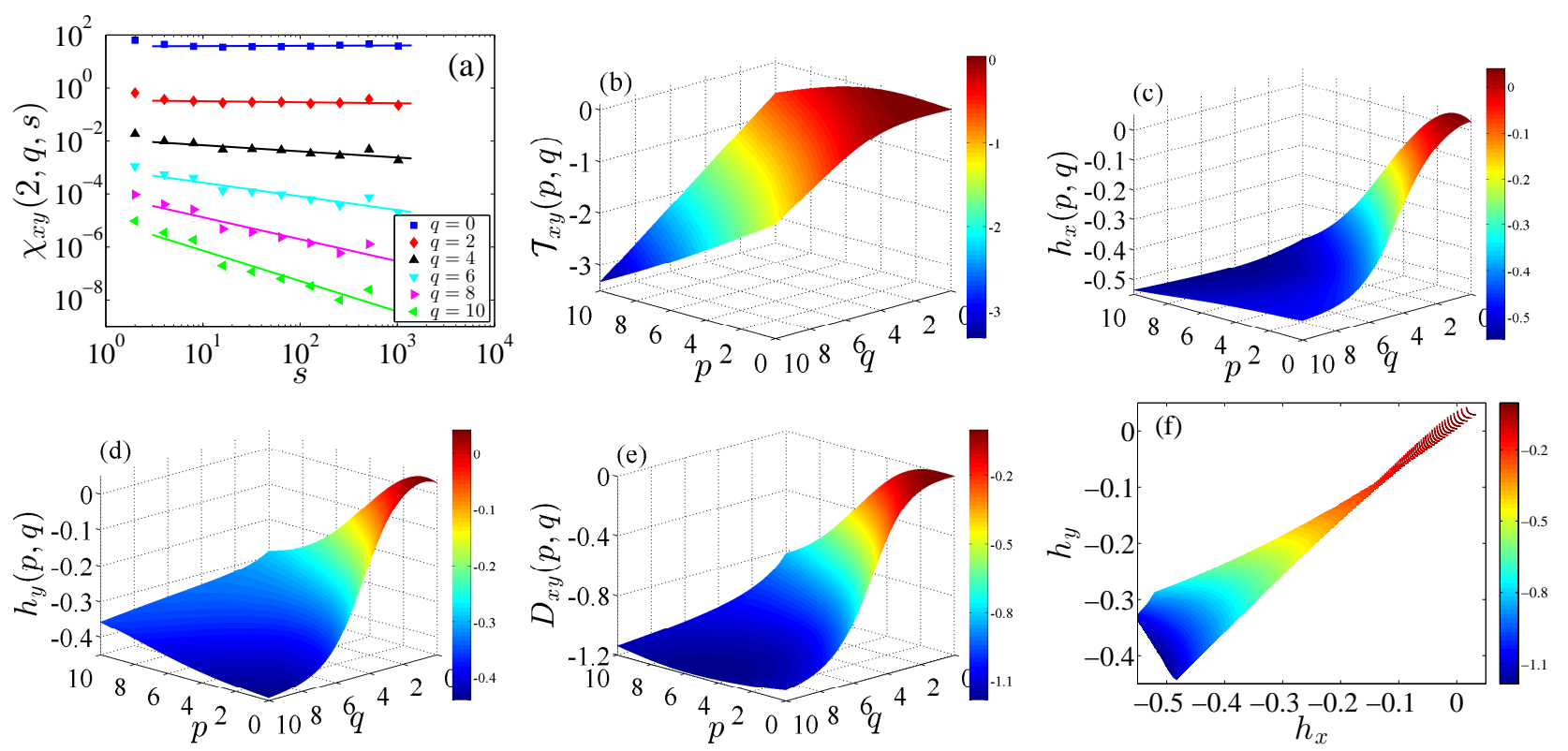

FIG. 6. (Color online) Multifractal cross wavelet analysis of the joint multifractality between the daily volatility series of DJIA index and NASDAQ index using the $\operatorname{MFXWT}(p, q)$ method. (a) Power-law dependence of $\chi_{x y}(p, q, s)$ on scale $s$ for fixed $p=2$ and different $q$. (b) Joint mass exponent function $\mathcal{T}(p, q)$. (c) Joint singularity strength function $h_{x}(p, q)$. (d) Joint singularity strength function $h_{y}(p, q)$. (e) Joint multifractal function $D_{x y}(p, q)$. (f) Joint multifractal singularity spectrum $D_{x y}\left(h_{x}, h_{y}\right)$.

Figures 5(c) and 5(d) show the joint singularity strength functions $h_{x}(p, q)$ and $h_{y}(p, q)$, which are numerically estimated from $\mathcal{T}(p, q)$. We find that both singularity strength functions are widely dispersed with spanning ranges greater than 0.3. In addition, the joint singularity strength functions are monotonic with respect to $p$ and $q$. Figure 5 (e) plots the joint multifractal function $D_{x y}(p, q)$ obtained from the double Legendre transform. Note that the joint multifractal function is located in the range of $(-1,0)$. The maximum point of $D_{x y}(p, q)$ is reached at $(p, q)=(0,0)$. Figure 5(f) shows the joint multifractal spectrum $D_{x y}\left(h_{x}, h_{y}\right)$, which does not collapse into the neighbor of a fixed point. Our empirical findings indicate that there is joint multifractality in the daily returns of the DJIA and the NASDAQ.

\section{B. Daily volatility time series}

We next use the $\operatorname{MFXWT}(p, q)$ method to perform a multifractal cross wavelet analysis of the daily volatilities of the two indices. The results are shown in Fig. 6 . 
Figure 6(a) shows a log-log plot of the dependence of the joint partition function $\chi_{x y}(2, q, s)$ with respect to the scale $s$ for fixed $p=2$ and varying $q$. We see strong power-law behaviors over two orders of magnitude. Figure 6(b) shows the resulting joint mass exponents $\mathcal{T}_{x y}(p, q)$, which are monotonically and nonlinearly increasing, implying that the cross correlations between the two index volatilities exhibit joint multifractality.

Figures 6(c) and 6(d) show the numerical calculations of the joint singularity strength functions $h_{x}(p, q)$ and $h_{y}(p, q)$, respectively. Note that the widths of both singularity strength functions are significantly larger than 0 , further confirming the existence of joint multifractality in the cross correlations of the two volatility time series. Figures 6(e) and 6(f) show the joint multifractal function $D_{x y}(p, q)$ and the joint multifractal spectrum $D_{x y}\left(h_{x}, h_{y}\right)$, respectively, which again affirms the joint multifractal characteristics in the cross correlations between the two index volatilities. Our results also show that the joint multifractal volatilities are stronger than those in the returns, because their widths of joint singularity strength functions and joint multifractal functions are larger. Our results are in accordance with the results of the cross multifractal analysis presented in Ref. [53], because the signs of returns will bring uncorrelated noise in comparison of pure volatilities.

\section{Origins of cross multifractality}

The fat-tailed distribution and linear and nonlinear long memory behaviors in financial series are considered as origins of multifractality [50, 54]. For cross multifractality, the memory behaviors may contain the following two constituents, the auto-correlation within each series and the cross correlation between series. Thus, it is interesting to investigate how these two types of correlation behaviors affect the cross multifractal nature. To implement the tests, we simply employ the width of multifractal spectrum for $p=q$ to quantitatively measure the degree of cross multifractality, which is defined by

$$
\Delta h_{x y}=\max \left[h_{x y}(p)\right]-\min \left[h_{x y}(p)\right]
$$

Such simplification is reasonable, as the spanning range of the diagonal line with $p=q$ of the $\mathcal{T}_{x y}$ surface approximately equals to the spanning range of the whole surface, evidenced by the surface plots of $\mathcal{T}_{x y}$ in Figs. 3.6 . We first test the effect of cross correlation behavior on the cross multifractality. Following Ref. [25], we shift two series from 1 day to 100 days relative to each other to gradually weaken the cross correlation between them without changing the auto-correlation in each series. Such a strategy also allows us to detect the possible time lags or asymmetry effects in cross multifractality.
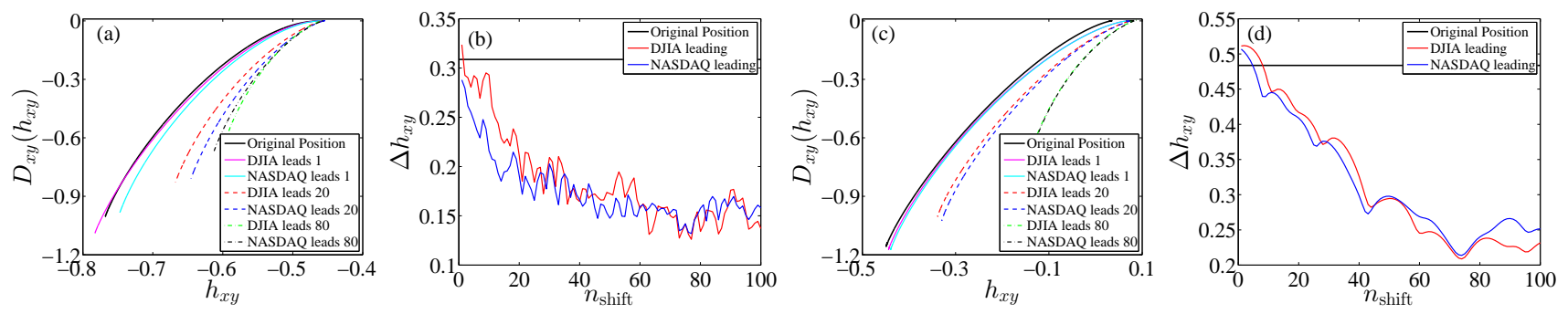

FIG. 7. (Color online) Results of cross multifractality obtained by gradually decreasing the strength of cross correlations, which is achieved by shifting two series relative to each other. (a) Cross multifractal spectra of the DJIA-NASDAQ returns with the original position, with the leading positions (1 day, 20 days and 80 days) for DJIA, and with the leading positions (1 day, 20 days, and 80 days) for NASDAQ. (b) Plots of the spectral width as a function of the number of shifted positions for returns. The spectral width of original returns is illustrated as the horizontal line. (c) The same as (a), but for volatilities. (d) The same as (b), but for volatilities.

For the DJIA-NASDAQ returns and volatilities, we estimate the multifractal spectra for three cases of positioning the time series relative to each other. The first case corresponds to no shifts between the two series. The second case is that DJIA is shifted relative to NASDAQ by $n_{\text {shift }}$ days in advance. The third case is that NASDAQ leads DJIA by $n_{\text {shift }}$ days. By setting $n_{\text {shift }}=1,10$, and 80 , we plot the obtained multifractal spectra in Fig. 7 (a) and (c) for returns and volatilities, respectively. We find that the pair of returns in which DJIA leads one day ahead of NASDAQ exhibit the strongest cross multifractality, as well as the pair of volatilities with DJIA leading one day ahead.

We further vary $n_{\text {shift }}$ from 1 to 100 and estimate the spectral width $\Delta h_{x y}$. The corresponding results of returns and volatilities are illustrated in Fig. 7 (b) and (d), in which $\Delta h_{x y}$ is plotted with respect to $n_{\text {shift }}$. We find that $\Delta h_{x y}$ decreases quickly with the increasing of $n_{\text {shift }}$, indicating the deterioration of the cross multifractality. This is due to that the cross correlation between two series becomes weaker when their lag increases, supporting that the 
cross correlation can be regarded as the origin of cross multifractality. In panels (b) and (d), another intriguing phenomenon is that for $n_{\text {shift }} \leq 30$ the multifractality of DJIA leading case is stronger than that of NASDAQ leading case, presenting that the influence of DJIA on NASDAQ in next few days is stronger than the influence of NASDAQ on DJIA. Such results also reveal that there is an asymmetry effect in cross correlation between DJIA and NASDAQ returns. We also find that $\Delta h_{x y}$ of volatilities is larger than that of returns, implying that the cross multifractality of volatilities is stronger than that of returns. As mentioned above, the sign of returns will introduce noise that can deteriorate the cross correlations comparing with volatilities [53].
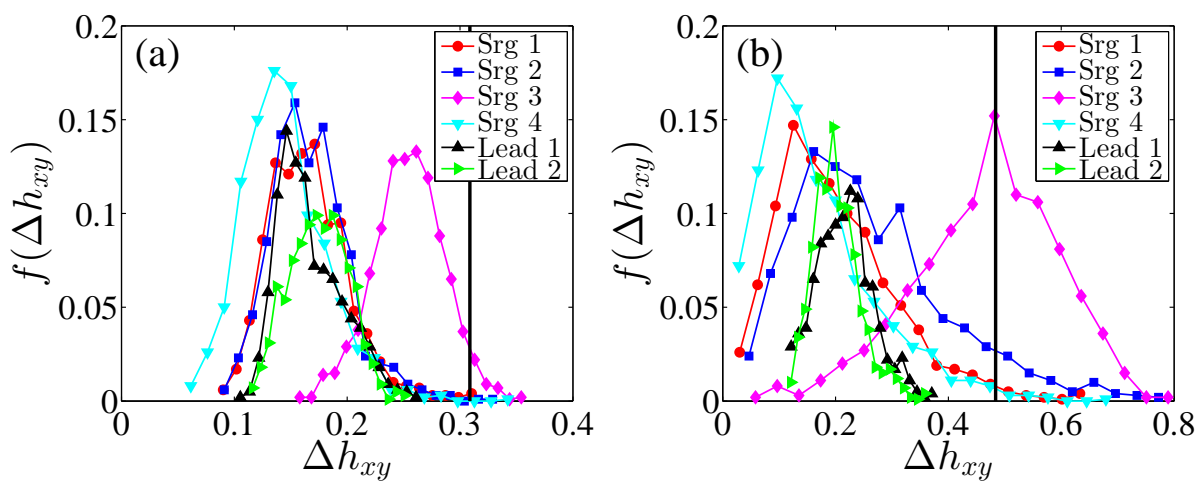

FIG. 8. (Color online) Distribution of the multifractal spectrum width for four surrogate pairs (labeled as Srgs 1-4) and two shifted pairs (labeled as Lead 1 and Lead 2). The spectral width of original pairs are shown in vertical black line. (a) DJIA-NASDAQ Returns. (b) DJIA-NASDAQ Volatilities.

We have shown that destroying the cross correlation between two series can strongly weaken the cross multifractality, which motivates us to further test how the auto-correlation in each series affects the cross multifractality. To conduct the tests, we generate four pairs of surrogate series, including (1) the shuffled DJIA and the original NASDAQ, (2) the original DJIA and the shuffled NASDAQ, (3) the co-shuffled DJIA and NASDAQ in which the data points of the two indexes on the same day are bounded as one single entity in the shuffling procedure, and (4) the shuffled DJIA and the shuffled NASDAQ in which the data points of each index are shuffled independently. The first surrogate pairs (termed as 'Srg 1') only preserve the auto-correlation in NASDAQ and destroy the auto-correlation in DJIA and the cross correlation between DJIA and NASDAQ. The second surrogate pairs (termed as 'Srg 2') only preserve the auto-correlation in DJIA and remove the auto-correlation in NASDAQ and the cross correlation between DJIA and NASDAQ. The third surrogate pairs (termed as 'Srg 3') remove the auto-correlation and cross correlation with non-zero lags, but keep the cross correlation with zero lag unchanged. In the the fourth surrogate pairs (termed as 'Srg 4'), all the possible correlations are removed. We generate 1000 synthetic pairs for each surrogate case, and perform the same wavelet cross multifractal analysis on each synthetic pair to estimate its multifractal spectrum width $\Delta h_{x y}$. We also employ two shifted pairs for comparison, One corresponding to DJIA leading NASDAQ (termed as 'Lead 1') and the other corresponding to NASDAQ leading DJIA (termed as 'Lead 2'). As shown in Fig. 7 (b) and (d), the width of multifractal spectrum is almost stable when the shifted position is greater than 80 , indicating that the cross correlation is completely removed when $n_{\text {shift }} \geq 80$. Thus, we set $n_{\text {shift }}$ varying from 101 to 1100 to generate 1000 pairs of synthetic data for each shifted pair. The shifted pairs here represent the surrogate data in which the cross correlation is removed and the auto-correlation remains unchanged.

Fig. 8 (a) and (b) illustrate the distributions of $\Delta h_{x y}$ obtained from four surrogate pairs and two shifted pairs for the DJTA-NASDAQ returns and volatilities. The vertical black line in both panels represents the spectral width of original data. Their average multifractal spectrum widths are reported in Table [ For DJIA-NASDAQ returns, we have the following inequality,

$$
\left\langle\Delta h_{x y}\right\rangle_{\text {Srg } 4}<\left\langle\Delta h_{x y}\right\rangle_{\text {Srg } 1} \approx\left\langle\Delta h_{x y}\right\rangle_{\text {Srg } 2} \approx\left\langle\Delta h_{x y}\right\rangle_{\text {Lead } 1} \approx\left\langle\Delta h_{x y}\right\rangle_{\text {Lead } 2}<\left\langle\Delta h_{x y}\right\rangle_{\text {Srg } 3}<\left\langle\Delta h_{x y}\right\rangle_{\text {Original }} .
$$

We can observe that the cross multifractal nature is the weakest if we remove both auto-correlations and cross correlations (Srg 4), indicating that both auto-correlation and cross correlation have influences on the cross multifractality. However, the auto-correlation has a very small influence, as the widths of the surrogate pairs, in which one series has the same auto-correlation as the original series ( $\operatorname{Srg} 1$ and $\operatorname{Srg} 2)$ and both series have the same auto-correlation as the original series (Lead 1 and Lead 2), are only slightly larger than the width of Srg 4. The distribution curves of $\Delta h_{x y}$ for $\operatorname{Srg} 1, \operatorname{Srg} 2$, Lead 1, and Lead 2 almost overlap with each other and their average values are all equal to 0.17 , far from the width of the original returns 0.31 , implying that the cross correlation plays an crucial role in the origin of cross multifractality. The cross correlation can be further decomposed into cross correlation with zero 
lag and with nonzero lag. By keeping the zero lag cross correlation unchanged and removing the nonzero lag cross correlation (Srg 3), we find that the obtained cross multifractal nature is the strongest in the surrogate experiments, suggesting that the zero lag cross correlation between returns contributes a great part on the cross multifractality. For DJIA-NASDAQ volatilities, we can obtain the following inequality,

$$
\left\langle\Delta h_{x y}\right\rangle_{\operatorname{Srg} 4}<\left\langle\Delta h_{x y}\right\rangle_{\operatorname{Srg} 1} \approx\left\langle\Delta h_{x y}\right\rangle_{\operatorname{Srg} 2} \approx\left\langle\Delta h_{x y}\right\rangle_{\text {Lead } 1} \approx\left\langle\Delta h_{x y}\right\rangle_{\text {Lead } 2}<\left\langle\Delta h_{x y}\right\rangle_{\text {Srg } 3} \approx\left\langle\Delta h_{x y}\right\rangle_{\text {Original }}
$$

Comparing with Eq. (24), the only difference is that $\left\langle\Delta h_{x y}\right\rangle_{\text {Srg } 3} \approx\left\langle\Delta h_{x y}\right\rangle_{\text {Original }}$ in Eq. 25, suggesting that the zero lag cross correlation between volatilities is the main origin of cross multifractality.

TABLE I. The average multifractal spectrum width of original pairs, four surrogate pairs, and two shifted pairs for DJIANASDAQ returns and volatilities.

\begin{tabular}{cccccccc}
\hline DJIA-NASDAQ & original & Srg 1 & Srg 2 & Srg 3 & Srg 4 & Lead 1 & Lead 2 \\
\hline Returns: $\left\langle\Delta h_{x y}\right\rangle$ & 0.31 & $0.17 \pm 0.04$ & $0.17 \pm 0.03$ & $0.25 \pm 0.03$ & $0.14 \pm 0.04$ & $0.17 \pm 0.03$ & $0.17 \pm 0.03$ \\
Volatilities: $\left\langle\Delta h_{x y}\right\rangle$ & 0.48 & $0.20 \pm 0.11$ & $0.26 \pm 0.14$ & $0.47 \pm 0.12$ & $0.17 \pm 0.10$ & $0.22 \pm 0.05$ & $0.20 \pm 0.04$ \\
\hline
\end{tabular}

\section{CONCLUSION AND DISCUSSION}

We have developed a new method of joint multifractal analysis with two moment orders based on wavelet transform, which we call $\operatorname{MFXWT}(p, q)$. Because some of the wavelet coefficients approach 0 , the values of $p$ and $q$ must be greater than 0 . We check the performance of the $\operatorname{MFXWT}(p, q)$ method using extensive numerical experiments on time series pairs generated from binomial measures and bivariate fractional Brownian motions. We also test the ability of this method to detect any joint multifractality in return pairs and volatility pairs in the US stock markets.

Using binomial measures from the $p$-model, we derive the theoretical expressions of the joint multifractality by comparing the scaling behaviors of the joint partition functions of the $\operatorname{MFXWT}(p, q)$ and the $\operatorname{MFXPF}(p, q)$ methods. We find that the joint multifractality $\left(\mathcal{T}_{x y}, h_{x}, h_{y}\right.$ and the $\left.D_{x y}\right)$ extracted using the MFXWT method closely agrees with theoretical values. This indicates that the accuracy of $\operatorname{MFXWT}(p, q)$ is sufficient to detect joint multifractality in binomial measures.

For bFBMs, we find that the joint mass exponent function $\mathcal{T}_{x y}$ of the cross correlations is linearly dependent on the orders $p$ and $q$, and this is a hallmark of monofractality. This clearly indicates the inherent monofractality in bFMBs. We find that the singularity strength functions $h_{x}$ and $h_{y}$ are in an extremely narrow range, which again confirms that bFMBs are monofractal. On the other hand, we are wary of the multifractal function $D_{x y}$ indicated by the MFXWT method, because this method yields biased outcomes for the bFBMs. We are also wary of the multifractality determined using the multifractal function $D_{x y}$ given by the MFXWT algorithm because it may indicate spurious multifractality, especially when we do not know a priori the underlying fractal properties. We can compensate for these shortcomings by performing statistical tests using the bootstrap method.

Unlike the $\operatorname{MFXPF}(p, q)$ method, which can be applied only to conservative measures (volatility), the MFXWT( $p, q$ ) method can analyze both conservative and non-conservative measures. We thus use it to analyze joint multifractality in the returns and volatilities of two US stock market indices. We find joint multifractality both in the returns and in the volatilities, and find that the joint multifractality in the volatilities is stronger than in the returns. We also find that the cross correlated behavior, particularly the zero lag cross correlation, is the main origin of cross multifractality.

The well-known shortcoming of the wavelet analysis of multifractals is that the moment order must be positive due to the presence of small wavelet coefficients, and thus because all the modulus maxima are significantly larger than 0 we must use the wavelet transform modulus maxima (WTMM) method [30, 36, 38, 55]. Unfortunately the WTMM method cannot be generalized to bivariate cases, because at each scale $s$ the number of modulus maxima of the two time series usually differ. 


\section{ACKNOWLEDGMENTS}

We acknowledge financial support from the National Natural Science Foundation of China (11375064, 71532009 and 71571121) and the Fundamental Research Funds for the Central Universities (222201718006).

[1] Wang, G.-J. \& Xie, C. Cross-correlations between the CSI 300 spot and futures markets. Nonlinear Dyn. 73, 1687-1896 (2013)

[2] Wang, D.-H., Suo, Y.-Y., Yu, X.-W. \& Lei, M. Price-volume cross-correlation analysis of CSI300 index futures. Physica A 392, 1172-1179 (2013).

[3] Ma, F., Wei, Y. \& Huang, D.-S. Multifractal detrended cross-correlation analysis between the Chinese stock market and surrounding stock markets. Physica A 392, 1659-1670 (2013).

[4] Zhou, Y. \& Chen, S. Cross-correlation analysis between Chinese TF contracts and treasury ETF based on high-frequency data. Physica A 443, 117-127 (2016).

[5] Wang, G.-J., Xie, C., He, L.-Y. \& Chen, S. Detrended minimum-variance hedge ratio: A new method for hedge ratio at different time scales. Physica A 405, 70-79 (2014).

[6] Chen, W., Wei, Y., Lang, Q.-Q., Lin, Y. \& Liu, M.-J. Financial market volatility and contagion effect: A copulaCmultifractal volatility approach. Physica A 398, 289-300 (2014).

[7] Meneveau, C., Sreenivasan, K. R., Kailasnath, P. \& Fan, M. S. Joint multifractal measures: Theory and applications to turbulence. Phys. Rev. A 41, 894-913 (1990).

[8] Xie, W.-J., Jiang, Z.-Q., Gu, G.-F., Xiong, X. \& Zhou, W.-X. Joint multifractal analysis based on the partition function approach: Analytical analysis, numerical simulations and empirical application. New J. Phys. 17, 103020 (2015).

[9] Wang, J., Shang, P. J. \& Ge, W. J. Multifractal cross-correlation analysis based on statistical moments. Fractals 20, 271-279 (2012).

[10] Kristoufek, L. Multifractal height cross-correlation analysis: A new method for analyzing long-range cross-correlations. EPL (Europhys. Lett.) 95, 68001 (2011).

[11] Barabási, A.-L. \& Vicsek, T. Multifractality of self-affine fractals. Phys. Rev. A 44, 2730-2733 (1991).

[12] Antonia, R. A. \& Van Atta, C. W. On the correlation between temperature and velocity dissipation fields in a heated turbulent jet. J. Fluid Mech. 67, 273-288 (1975).

[13] Zhou, W.-X. Multifractal detrended cross-correlation analysis for two nonstationary signals. Phys. Rev. E 77, 066211 (2008).

[14] Podobnik, B. \& Stanley, H. E. Detrended cross-correlation analysis: A new method for analyzing two nonstationary time series. Phys. Rev. Lett. 100, 084102 (2008).

[15] Jiang, Z.-Q. \& Zhou, W.-X. Multifractal detrending moving-average cross-correlation analysis. Phys. Rev. E 84, 016106 (2011).

[16] Gu, G.-F. \& Zhou, W.-X. Detrending moving average algorithm for multifractals. Phys. Rev. E 82, 011136 (2010).

[17] Alessio, E., Carbone, A., Castelli, G. \& Frappietro, V. Second-order moving average and scaling of stochastic time series. Eur. Phys. J. B 27, 197-200 (2002).

[18] Carbone, A. \& Castelli, G. Scaling properties of long-range correlated noisy signals: Appplication to financial markets. Proc. SPIE 5114, 406-414 (2003).

[19] Carbone, A., Castelli, G. \& Stanley, H. E. Analysis of clusters formed by the moving average of a long-range correlated time series. Phys. Rev. E 69, 026105 (2004).

[20] Arianos, S. \& Carbone, A. Detrending moving average algorithm: A closed-form approximation of the scaling law. Physica A 382, 9-15 (2007).

[21] Carbone, A. Algorithm to estimate the Hurst exponent of high-dimensional fractals. Phys. Rev. E 76, 056703 (2007).

[22] Carbone, A. \& Kiyono, K. Detrending moving average algorithm: Frequency response and scaling performances. Phys. Rev. E 93, 063309 (2016).

[23] Tsujimoto, Y., Miki, Y., Shimatani, S. \& Kiyono, K. Fast algorithm for scaling analysis with higher-order detrending moving average method. Phys. Rev. E 93, 053304 (2016).

[24] Kiyono, K. \& Tsujimoto, Y. Time and frequency domain characteristics of detrending-operation-based scaling analysis: Exact DFA and DMA frequency responses. Phys. Rev. E 94, 012111 (2016).

[25] Oświęcimka, P., Drożdż, S., Forczek, M., Jadach, S. \& Kwapień, J. Detrended cross-correlation analysis consistently extended to multifractality. Phys. Rev. E 89, 023305 (2014).

[26] Kwapień, J., Oświęcimka, P. \& Drożdż, S. Detrended fluctuation analysis made flexible to detect range of cross-correlated fluctuations. Phys. Rev. E 92, 052815 (2015).

[27] Qian, X.-Y. et al. Detrended partial cross-correlation analysis of two nonstationary time series influenced by common external forces. Phys. Rev. E 91, 062816 (2015).

[28] Holschneider, M. On the wavelet transformation of fractal objects. J. Stat. Phys. 50, 963-993 (1988).

[29] Arneodo, A., Grasseau, G. \& Holschneider, M. Wavelet transform of multifractals. Phys. Rev. Lett. 61, 2281-2284 (1988).

[30] Muzy, J. F., Bacry, E. \& Arnéodo, A. Wavelets and multifractal formalism for singular signals: Application to turbulence data. Phys. Rev. Lett. 67, 3515-3518 (1991). 
[31] Hudgins, L., Friehe, C. A. \& Mayer, M. E. Wavelet transforms and atmospheric turbulence. Phys. Rev. Lett. 71, 3279-3282 (1993).

[32] Maraun, D. \& Kurths, J. Cross wavelet analysis: significance testing and pitfalls. Nonlin. Process. Geophys. 11, 505-514 (2004).

[33] Aguiar-Conraria, L. \& Soares, M. J. The continuous wavelet transform: Moving beyond uni- and bivariate analysis. J. Econ. Surv. 28, 344-375 (2014).

[34] Kantelhardt, J. W. et al. Multifractal detrended fluctuation analysis of nonstationary time series. Physica A 316, 87-114 (2002).

[35] Oświęcimka, P., Kwapień, J. \& Drożdż, S. Wavelet versus detrended fluctuation analysis of multifractal structures. Phys. Rev. E 74, 016103 (2006).

[36] Bacry, E., Muzy, J. F. \& Arnéodo, A. Singularity spectrum of fractal signals from wavelet analysis: Exact results. J. Stat. Phys. 70, 635-674 (1993).

[37] Audit, B., Bacry, E., Muzy, J.-F. \& Arnéodo, A. Wavelet-based estimators of scaling behavior. IEEE Trans. Info. Theory 48, 2938-2954 (2002).

[38] Muzy, J. F., Bacry, E. \& Arnéodo, A. The multifractal formalism revisited with wavelets. Int. J. Bifurcation Chaos 4, 245-302 (1994).

[39] Grinsted, A., Moore, J. C. \& Jevrejeva, S. Application of the cross wavelet transform and wavelet coherence to geophysical time series. Nonlin. Process. Geophys. 11, 561-566 (2004).

[40] Rua, A. \& Nunes, L. C. International comovement of stock market returns: A wavelet analysis. J. Empir. Financ. 16, 632-639 (2009).

[41] Vacha, L. \& Barunik, J. Co-movement of energy commodities revisited: Evidence from wavelet coherence analysis. Energy Econ. 34, 241-247 (2011).

[42] Meneveau, C. \& Sreenivasan, K. R. Simple multifractal cascade model for fully developed turbulence. Phys. Rev. Lett. 59, 1424-1427 (1987).

[43] Lavancier, F., Philippe, A. \& Surgailis, D. Covariance function of vector self-similar processes. Statist. Prob. Lett. 79, 2415-2421 (2009).

[44] Coeurjolly, J.-F., Amblard, P.-O. \& Achard, S. On multivariate fractional Brownian motion and multivariate fractional Gaussian noise. Eur. Signal Process. Conf. 18, 1567-1571 (2010).

[45] Amblard, P.-O., Coeurjolly, J.-F., Lavancier, F. \& Philippe, A. Basic properties of the multivariate fractional Brownian motion. Bulletin Soc. Math. France, Séminaires et Congrès 28, 65-87 (2013).

[46] Halsey, T. C., Jensen, M. H., Kadanoff, L. P., Procaccia, I. \& Shraiman, B. I. Fractal measures and their singularities: The characterization of strange sets. Phys. Rev. A 33, 1141-1151 (1986).

[47] Turiel, A. \& Parga, N. The multifractal structure of contrast changes in natural images: From sharp edges to textures. Neural Comput. 12, 763-793 (2000).

[48] Kestener, P. \& Arneodo, A. Three-dimensional wavelet-based multifractal method: The need for revisiting the multifractal description of turbulence dissipation data. Phys. Rev. Lett. 91, 194501 (2003).

[49] Turiel, A., Perez-Vicente, C. J. \& Grazzini, J. Numerical methods for the estimation of multifractal singularity spectra on sampled data: A comparative study. J. Comput. Phys. 216, 362-390 (2006).

[50] Zhou, W.-X. Finite-size effect and the components of multifractality in financial volatility. Chaos Solitons Fractals 45, $147-155(2012)$.

[51] Jiang, Z.-Q. \& Zhou, W.-X. Scale invariant distribution and multifractality of volatility multipliers in stock markets. Physica A 381, 343-350 (2007).

[52] Jiang, Z.-Q., Xie, W.-J. \& Zhou, W.-X. Testing the weak-form efficiency of the WTI crude oil futures market. Physica A 405, 235-244 (2014).

[53] Rak, R., Drożdż, S., Kwapień, J. \& Oswiecimka, P. Detrended cross-correlations between returns, volatility, trading activity, and volume traded for the stock market companies. EPL (Europhys. Lett.) 112, 48001 (2015).

[54] Zhou, W.-X. The components of empirical multifractality in financial returns. EPL (Europhys. Lett.) 88, 28004 (2009).

[55] Muzy, J. F., Bacry, E. \& Arnéodo, A. Multifractal formalism for fractal signals: The structure-function approach versus the wavelet-transform modulus-maxima method. Phys. Rev. E 47, 875-884 (1993). 